\title{
Prevalence of central nervous system adverse effect and associated factors among HIV patients on efavirenz based ART regimen
}

Esileman Abdela Muche ( $\sim$ SulamanAbdela@gmail.com )

University of Gondar College of Medicine and Health Sciences

Mekdes Kiflu

\section{Research}

Keywords: Central nervous system adverse effect, University of Gondar compressive and specialized hospital, Efavirenz based ART regimen

Posted Date: February 18th, 2020

DOI: https://doi.org/10.21203/rs.2.23835/v1

License: (1) This work is licensed under a Creative Commons Attribution 4.0 International License. Read Full License 


\section{Abstract}

Background: Efavirenz based antiretroviral regimens served as a first line treatment in our country, however commonly lead to central nervous system toxicity, which is the main reason for non-adherence, switch and discontinuation to antiretroviral therapy. We aimed to assess prevalence of central nervous system adverse effect and associated factors among HIV patients taking efavirenz based regimens.

Methods: Hospital based cross-sectional study was conducted on 345 patients who were taking efavirenz based ART medications on follow up at UoGCSH from March 15 to may15, 2018. Information on sociodemographic characteristics and detailed history regarding central nervous system adverse effect were taken from both the patient and medical record.. Both bivariate and multivariate analyses were done using binary logistic regression to find out the associated factors. Level of statistical significance was declared at $P$ value of $\leq 0.05$ levels.

Result: The central nervous system adverse effects of EFV based ART regimen were observed in $52.8 \%$ of participants and vivid dream was the most commonly reported adverse effect. Upon computation of bivariate binary logistic regression economic status, EFV based regimen type, Stage of RVI, alcohol use and concomitant medical condition had shown association on the occurrence of central nervous system adverse effect of EFV based ART regimen. But in multivariate analysis alcohol use $(p=0.004 ; A O R=$ 4.450), stage I RVI $(p=0.001 ; A O R=0.006)$ stage II RVI $(p=0.001 ; A O R=0.017)$ stage III RVI $(P=0.01$; $A O R=4.13$ ) became significantly associated with the occurrence of CNS adverse effect.

Conclusion: More than half of HIV patients taking efavirenz based regimens at UOGCSH experience the central nervous system adverse effect. The most commonly reported central nervous system adverse effects were vivid dream, confusion, and insomnia. Stage III and IV HIV patient need special attention to prevent and decrease central nervous system adverse effect of EFV based regimen. Health workers need to tell the patients to avoid alcohol, especially in the initiation of the treatment.

\section{Introduction}

The first HIV case in Ethiopia was detected in 1984. The 2014 ART need is 542121 for adults and 178500 for children under 15 years of age.(1) Antiretroviral therapy have the capacity of reducing the viral load to the undetectable level, reduce HIV related opportunistic infection by increasing the T-lymphocyte, CD4 counts and radical decrement in morbidity and mortality. Beside to their benefit they might cause adverse reaction which is the main reason for drug discontinuation, switch and non-adherence to antiretroviral therapy. This adverse drug reaction consequently leads to treatment failure. (2-4)

The magnitude and usually nature of highly active antiretroviral therapy associated adverse effects have contributed to the current re-thinking and debate about ART. Evidences showed that prevalence of adverse effects were much higher in developing countries than in developed countries (USA 66.7\%, India 71.11\%, South Africa $47 \%$, and Ethiopia $88 \%$ ). So Ethiopia is the most highly affected in adverse effects when compared to other countries.(5) 
Efavirenz is a potent and effective non- nucleoside reverse transcriptase inhibitor and has an activity against HIV- 1 that has become a backbone of antiretroviral regimens in combination with other drugs. (6, 7) Efavirez has prolonged half-life allowing once-daily regimens. $(8,9)$ Nucleoside reverse transcriptase inhibitor comprising TDF + 3TC as backbone and one non- nucleoside reverse transcriptase inhibitor (EFV) are maintained as the preferred choices in all patients older than ten years because of its high viral superesession capacity it serves as a first-line treatment; however adverse central-nervous and neuropsychiatric side effects are frequently reported. $(10,11)$

Efavirenz compare to structurally similar agent, nevirapine, neuropsychiatric effects occur more frequently and with greater severity, but the reason is unknown.(12) Concerns about the use of efavirenz in patients with a history of mental illness and psychiatric diseases may predispose clinicians not to offer this agent in spite of the convenience of once daily dosing, which can result in improved adherence in these at-risk populations.(13)

EFV treated patients experience more side effect and the risk of discontinuation of treatment as a result of experiencing symptoms was higher in efavirenz treated patients compared non efavirenz based regimen. (14) Fifty four percent of patients on efavirenz had at least one neuropsychiatric disorder most of the time it appear within 4 weeks.(15)

The study done at Ambo zonal hospital in Ethiopia showed that occurrence of CNS adverse effect was $35.7 \%$, which is the second most frequent side effect. Low BMI and CD4 count, presence of co morbidities and treated by TDF/3-TC/EFV based regimen were the major risk factor for the occurrence of side effect.

Neuropsychiatric disorders are the most common and significant side effects associated with EFV therapy. CNS side effect reduce the patient quality of life which leads to loss of confidence on the health care and the drug, which makes the patient non adherent to the medication finally enhance reduction of the drug outcome and cause treatment failure This side effect makes the patient non-adherent to the medication and discontinuation of the drug ranging from $4-46 \%$. $(14,17,18)$

Eventhough differente researchs were conducted in the world especially in Africa there is a difference in the occurrences of CNS adverse effect of efaverenze due to genetic and socio demographic factors so it need relevant epidemiological studies to inform programmatic decisions given the shift towards EFVcontaining regimens for the management of HIV infection. Currently there is no sufficient data exists in UoGCSH and in Ethiopia on the prevalence of such CNS adverse effect specifically following the use of the antiretroviral therapy containing efavirenz. Most researches done in Ethiopia are focused on the general side effect of efavirenz not specifically for CNS and they did not assess the associated factors. So, the aim of this study is to assess the prevalence of Central nervous system adverse effect and identifying the possible contributing factors.

\section{Method}




\subsection{Study Area And Period}

The study was conducted at University of Gondar comprehensive and specialized Hospital ART clinic, Gondar town, northwest Ethiopia. It is located 737 kilometers away from the capital city of Ethiopia, Addis abeba; it is one of the biggest tertiary level referral and teaching hospital in the region. It provides a service of an estimated 7 million population. The hospital serves as a referral center for North Gondar administrative region and the residents around. It has 5 pharmacy units; one of the pharmacy units which serve many patients is ART pharmacy. This reseach was conducted from March 15 to may15, 2018.

\subsection{Study Design}

- Hospital based cross-sectional study was conducted.

\subsection{Source And Study Population}

- All HIV patients on EFV based regimen attending ART pharmacy at UOGCSH were considered as source population.

- The study population comprised of adult HIV positive patients on an Efavirenz based regimen who visited the ART pharmacy between March 15, 2018 and May, 15, 2018, at UOGCSH.

\subsection{Inclusion and exclusion criteria}

\section{i. Inclusion criteria:}

- Adult patients on an Efavirenz based regimen who gave their consent to take part in the study.

- Age greater than 15 years

\section{ii. Exclusion criteria:}

- Pregnant women

- Patients with documented CNS illness prior to starting Efavirenz.

- adolesents who is not

- patients with psychotic problem.

\subsection{Sample Size \& Sampling Methods}

The sample size was calculated using the following single population proportion formula, where 1.96 used for $Z a / 2,50 \%$ for $P$ and $5 \%$ for $d$ because there is no previous study in the study area. Where: $d$ is margin of error; $p$ is proportion of treatment outcome and $Z a / 2$ is the $95 \%$ confidence interval. 


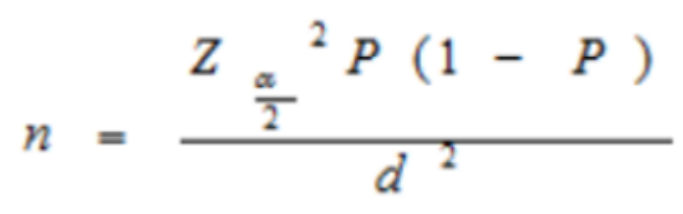

The total sample size required for the study was 384 . Since the total population is $<10,000$ the sample will be recalculated based on the following reduction formula;

$N f=n /(1+(n / N))$

$\mathrm{n}=$ sample size calculated by using population $>10,000, \mathrm{Nf}=$ actual sample size

$\mathrm{N}=$ total number of a patients taking efaverenze based regimen in ART pharmacy at UOGCSH, $(\mathrm{N}=1900)$. Therefore, the sample size was: $\mathrm{Nf}=384 / 1+384 / 1900=319$

By adding $8 \%$ contingency, a total of 345 patients were sampled. Patients were selected by using convenient sampling method at which the patients whose follow up appointment date was between March 15 and May15, 2018

\subsection{Data collection procedure}

Data was extracted by interviewing the patient using well-structured questionnaire and by reviewing the patient's medical chart. Patients were selected by using simple random sampling method at which the patients whose follow up appointment date was between March 15 and May15, 2018 were interviewed by using a structured questionnaire, after being taken through the informed consent form and on sending to take part in the study, additionally the patient chart was also reviewed.

\subsection{Quality Assurance}

Data was taken from patients' medical chart and patient interview using data extraction format; which was pretested in 17 patients ( $5 \%$ of calculated sample size) in UOGCSH. A Little adjustment was made to clarify questions. Filled document revised and double-checked during data entry by investigators.

\subsection{Variables}

\subsubsection{Independent variables}

- Demographic variables (age, sex, educational qualification, economic statuse, marital status, occupation, no of children,) 
- Patient clinical characteristics (type and duration of treatment with EFV, stage of the diseases, immunity status, comorbid conditions and concomitant medication use)

\subsubsection{Dependent variables}

- Central nervous system averse effect.

\subsection{Ethical Consideration}

Letter of ethical clearance was obtained from an ethical review committee of school of pharmacy. Written consent and letter of cooperation were obtained from University of Gondar referral hospital before going to chronic outpatient department. Written consent was taken from patients before starting the interview. Privacy and confidentiality were ensured during patient interview and review of patients' chart. Thus, name and address of the patient were not recorded in the data extraction formats. Each patient was informed about the objective of the study, procedures for selection and assurance of confidentiality. The right of the respondents for being interviewed was respected and this did not affect the service they get from the hospital.

\section{Result}

From the total of 345 patients included more than half of the pateint were male (59.7\%), almost one third of the participant attained secondary school $(33.5 \%)$ and majority of patient $(70.9 \%)$ were orthodox in religion. Most of the participant had lower economic status (71.7), large proportion of the patient were single (36.5\%) and unemployed (35.1\%). Analysis on number of children per household revealed that most of the household had $<4$ children $(76.6 \%)$. Table 1 shows the participants socio-demographic characteristics.

Almost two-third of the patients had normal BMI (69.9\%) and more than one third of participants had $<200$ cells/mm3 CD4 count (35.4\%). Two hundred eighty-four patients did not have prior medical condition and more than half of participants were in advanced stage $(55 \%)$.

Higher proportion of the patients were on TDF+3TC+EFV based regimen $(78.9 \%)$ and $73.1 \%$ were taking the medication at night and $67.6 \%$ were taking the drug after meal. Majority of the patients were taking the drug for 1-3 years (34.7\%) and no one was taking narcotics and also the most commonly used medication other than ART were contraceptives $14.2 \%$.

The overall occurrence of the CNS adverse effect was $52.8 \%$ and the most reported CNS side effect was vivid dream $29.8 \%$ followed by confusion $27.7 \%$, insomnia $24 \%$, somnolence or drowsiness $20.8 \%$, vertigo $8.7 \%$, impaired concentration $6 \%$, hallucination $3.5 \%$ and psychosis $3.2 \%$. About $35 \%$ of the participant resolved the side effect within 1-4 weeks. The detailed clinical characteristics of participants presented in table 2. 
The logistic regression analysis result showed in table3 there were no significant association between gender $(P=0.321)$, age $(P=0.651)$, education $(P=0.476)$, religion $(P=0.742)$, marital status $(P=0.441)$ employment status $(p=0.802)$, number of children $(p=0.501)$ with the occurrence of central nervous system adverse effect. In health status of the pateint prior medical condition ( $P=0.94)$, concurent use of other medication ( $P=1.0)$, time of the day medication is taken $(P=0.741)$, use of the drug with meal $(P=0.869)$, concomitant use of cotrimoxazole and isonoizide $(P=0.905)$ had no sinificant association. But there was association between CNS adverse effect occurance and economic status $(p<0.001)$, TDF+3TC+EFV based regimen type $(p<0.001$ COR=0.234), Stage of RVI $(p=0.01)$ Alcohol use $(p=0.07$ $\mathrm{COR}=0.677)$ and Concomitant medical condition $(p=0.08 \mathrm{COR}=1.85)$. Finally, in multivariate analysis alcohol use $(p=0.004$ AOR $=4.450)$, stage I RVI $(p=0.001, A O R=0.006)$ stage II RVI $(p=0.001$, $A O R=0.017)$ stage III RVI $(P=0.01, A O R=0.01)$ become significantly associated with the occurrence of central nervous system adverse effect. Table 3 shows the factors associated with efavirenz CNS adverse effect.

\section{Discussion}

In our study the prevalence of Efavirenz based regimen induced central nervous system adverse effect was $182(52.8 \%)$, which is similar with other research done in Kenya and America. $(16,19)$ But higher than Ambo referral hospital study (35.7\%) The difference might be due to Ambo study include only review of patient charts, include patients who takes the drug for more than 6 months, and include patients on non efavirenz based ART regimen.

The most commonly reported CNS adverse effect in our study was vivid dreams (29.5\%), confusion (23.4\%), insomnia (23.3\%), somnolence (21\%), and headache (19.9\%) but Kenyan study found that the most common CNS adverse effect was vertigo (dizziness) (25.5\%), nightmares (13.6\%), somnolence (drowsiness) (9.5\%) and insomnia was the least (1\%) this might be due to difference in sociodemographic and genetic difference among the participants.(16) But similar with the research done in Ghana, Ethiopia and Uganda $(13,20,21$,$) at which headache and insomnia were the most prevalent$ neuropsychiatric adverse effect.

In our research Age, gender, economic status, marital status, employment status and number of children, were not significantly associated with the occurrence of CNS side effect in multivariate analysis. Economic status was associated with the occurrence of CNS ADRs during treatment with an efavirenz based regimen in bivariate analysis, but when carrying out multivariate analysis it became insignificant. This finding is not in support of different studies in the world. (22-26)

Most patients were at the age of 25-45 years in this study which was similar with the research done in Nigeria which identified young adults in the productive age of 25 to 45 years (78\%) were those mostly infected with HIV and accessing ART. This finding was presented with a 2015 report reflected this age group as the most at risk of HIV infection. (27) 
Variables with $p$ values less than 0.3 during bivariate analysis entered into the multivariate analysis like economic status, efavirenz based regimen, stage, concomitant medical condition, alcohol use but only alcohol use and stage became significantly associated. BMI did not have association with the occurrence of CNS side effect in our study. More than half of the patient who experience the adverse effect was on BMI range (18.5-25) and, but other studies shown that BMI enhance the incidence of the CNS adverse effect. $(12,28,16)$

As TDF + 3TC + EFV serves as the first line regimen in Ethiopia $89.6 \%$ of patients who experienced CNS adverse effect took this regimen and remaining took AZT + 3TC + EFV $(10.4 \%)$, which showed no association with the occurrence of CNS adverse effect which is similar with Kenyan research. (16) But different from Ambo referral hospital study in Ethiopia and Nigerian study. $(12,23)$ The difference might be sample size and the method difference, because the data only comes from chart review, so it is more susceptible to bias.(12)

In our research only alcohol use and stage of disease became significantly associated with the occurrence of CNS adverse effect. stage I RVI $(p=0.001$, AOR $=0.006)$ which means stage I RVI patients were $99.4 \%$ less likely to experience CNS adverse effect than stage IV RVI patient. Stage II RVI $(p=0.001$, AOR $=0.017$ ) can be interpreted as $98.3 \%$ less likely to experience CNS side effect than stage IV patients. Stage III RVI $(P=0.01, A O R=4.13)$ which showed patients in stage III experienced 4.13 times more CNS side effect than stage IV patients. the least reaction was seen in stage 1 which was in line with what was found in Bahr Dare Felege- Hiwot referral hospital at which The risks of ADRs in WHO clinical stage II, III and IV were much higher than in Stage 1. (29) Most ADR were occurred in stage1 and 2 according to the research done in Nigeria the difference might be due to hence some clinical side effect may overlap with symptoms of HIV/AIDS in symptomatic patients (WHO stage 3 or 4) and might be missed, potentially resulting in under-reporting of adverse drug reactions in symptomatic patients. (30)

In this study tuberclosis (17.3\%) is the leading medical condition happened prior to starting the ART and the majority of the patient didn't have prior medical condition (82.7\%). There is no statistically significant asociation of the occurance of CNS adverse effect with prior medical condition but Nigerian research shown high proportion of medical condition is accounted by tuerclosis then followed by meningitis, and pnemonia had association with the occurrence of side effect. The difference observed was due to ahigh occurance of infectious disease in Nigeria comapared to Ethiopia. (3)

Analysis of socio-behavioral characteristics among the respondents revealed that a higher proportion (39.9\%) used alcohol and few participants smoked cigarettes (8.1\%) whereas no one used narcotic drugs. Smoking didn't have significant effect on the occurrence of CNS adverse effect but alcohol use significantly associated with the occurrence of CNS adverse effect $(p=0.004$ AOR $=4.450)$ which means patients who drink alcohol had 4.45 times more likely to encounter CNS adverse effect than patients who do not drink alcohol.

Limitation of the study 
Patient may experience recall bias and being cross sectional study was difficult to confirm cause and effect of the CNS adverse effect and Efavirenz and, additionally it is single center study, so generalization for other area may be difficult.

\section{Conclusion}

More than half of RVI patients taking Efavirenz based regimens at UOGCSH experienced the CNS adverse effect. Vivid dream, confusion, and insomnia were the most commonly reported CNS side effect. Most of the CNS side effect experienced by the respondents occurred within the 1st year of treatment and resolved 1-4 weeks after the initiation of the therapy. Alcohol use and advanced stage at the time of initiation of EFV was significantly associated with the incidence of CNS side effect.

\section{Recommendation}

- Advanced stage patients need close monitoring during initiation of the treatment by health workers.

- Health workers need to tell the patients to avoid alcohol, especially in the initiation of the treatment

\section{List Of Abbreviations}

ADR-Adverse Drug Reaction

AOR-Adjusted odd ratio

ART: Antiretroviral therapy

BMI- Body mass index

CD4 -T helper cells that carry the CD4 glycoprotein on their surface

CNS- Central Nervous System

COR- Crude odd ratio

EFV- Efavirenz

SPSS- Statistical package for social science

TDF-Tenofovir

WHO- World health organization

UoGCSH-University of Gondar compressive specialized hospital

3TC-Lamivudine 


\section{Declarations}

Acknowledgments: I would like to thank the University of Gondar for cooperation to do this research project. I am also very grateful to the nursing staffs of the ART clinic and Pharmacy of UoGRH for their cooperation in the data collection process. Finally, I would like to thank Rahawa Kiyar for her continuous advice and participate in data collection process of this work.

Ethics approval and consent to participate: Ethical clearance was obtained from the Ethical Review Committee of School of Pharmacy, University of Gondar. The respondents were informed about the purpose of the study and their consent to participate was obtained.

Consent to publish: participants consent was taken to publish this work

Availability of data and materials: The datasets during and/or analyzed during the current study is available from the author on reasonable request.

Funding: no funding source.

Competing interests: The author reports no conflicts of interest in this work.

Authors contribution: Both authors contributed in all component of the research.

\section{References}

1. Minstry of Health. National guidelines for comprehensive hiv prevention , care and treatment. 2017; Accesssed at https://aidsfree.usaid.gov/sites/default/files/resources/ethiopia_art_guidelines_2017

2. Masenyetse LJ, Manda SO, Mwambi HG. An assessment of adverse drug reactions among HIV positive patients receiving antiretroviral treatment in South Africa. AIDS research and therapy. 2015 Dec;12(1):6.

3. Agu $\mathrm{K}$, Ochei $\mathrm{U}$, Oparah A, Onoh $\mathrm{O}$. Treatment outcomes in patients receiving combination antiretroviral therapy in Central Hospital, Benin City, Nigeria. Tropical Journal of Pharmaceutical Research. 2010; 9 (1).

4. Agu KA, RC K, MA I, PG I. Medication Adherence and Risk factors for non-adherence among patients taking Highly Active Antiretroviral Therapy. West African Journal of Pharmacy. 2011 Mar 13; 22 (1).

5. Li H, Marley G, Ma W, Wei C, Lackey M, Ma Q, Renaud F, Vitoria M, Beanland R, Doherty M, Tucker JD. The role of ARV associated adverse drug reactions in influencing adherence among HIV-infected individuals: a systematic review and qualitative meta-synthesis. AIDS and Behavior. 2017 Feb 1;21(2):341-51.

6. Mazzotta E, Agostinone A, Rosso R, Di Biagio A, De Socio GV, Cappelletti A, Zicolella R, Polilli E, Bonfanti P, Di Matteo L, Manzoli L. Osteonecrosis in human immunodeficiency virus (HIV)-infected patients: a multicentric case-control study. Journal of bone and mineral metabolism. 2011 May 1;29(3):383-8. 
7. Aberg JA. JIAPAC. Lo I. Notes \& Comments. 2003;160-1.

8. Marzolini C, Telenti A, Decosterd LA, Greub G, Biollaz J, Buclin T. Efavirenz plasma levels can predict treatment failure and central nervous system side effects in HIV-1-infected patients. Aids. 2001 Jan 5;15(1):71-5.

9. Adkins JC, Noble S. Efavirenz. Drugs. 1998 Dec 1;56(6):1055-64. https://doi.org/10.2165/00003495$199856060-00014$

10. Joint UN. Programme on HIV/AIDS. Global AIDS Update 2016. Joint United Nations Programme on HIV. AIDS. 2016.

11. Teklay G, Legesse B, Legesse M (2013) Adverse Effects and Regimen Switch among Patients on Antiretroviral Treatment in a Resource Limited Setting in Ethiopia. J Pharmacovigilance 1: 115. doi:10.4172/2329-6887.1000115

12. IJBCP International Journal of Basic \& Clinical Pharmacology Assessment of ART adverse reactions and determinants at primary hospital in Ethiopia. 2013;2(2):208-15.

13. Kenedi CA, Goforth HW. A systematic review of the psychiatric side-effects of efavirenz. AIDS and Behavior. 2011 Nov 1;15(8):1803-18.

14. Fumaz CR, Munoz-Moreno JA, Moltó J, Negredo E, Ferrer MJ, Sirera G, Pérez-Alvarez N, Gómez G, Burger D, Clotet B. Long-term neuropsychiatric disorders on efavirenz-based approaches: quality of life, psychologic issues, and adherence. JAIDS Journal of Acquired Immune Deficiency Syndromes. 2005 Apr 15;38(5):560-5.

15. Padilla S, Anto R, Masia M, Martı A. Prediction of Neuropsychiatric Adverse Events Associated with Long-Term Efavirenz Therapy, Using Plasma Drug Level Monitoring. 2018;41(May):0-5.

16. Wambura CA. Assessment of the Prevalence of Central Nervous System Adverse Reactions in adult patients taking Efavirenz based regimens at Mbagathi District Hospital's comprehensive Care Centre (Doctoral dissertation, JKUAT).2015;

17. Abers MS, Shandera WX, Kass JS. Neurological and psychiatric adverse effects of antiretroviral drugs. CNS drugs. 2014 Feb 1;28(2):131-45.

18. Mehta U, Durrheim DN, Blockman M, Kredo T, Gounden R, Barnes KI. Adverse drug reactions in adult medical inpatients in a South African hospital serving a community with a high HIV/AIDS prevalence: prospective observational study. British journal of clinical pharmacology. 2008 Mar;65(3):396-406.

19. Mueller TE, Ellwanger JH, Michita RT, Matte MC, Renner JD. CYP2B6 516 G> T polymorphism and side effects of the central nervous system in HIV-positive individuals under Efavirenz treatment: Study of a sample from southern Brazil. Anais da Academia Brasileira de Ciências. 2017 May;89(1):497-504.

20. Celesia BM, Sofia SA, Rapisarda L, Maresca M, Vinci L, Gussio M, et al. Anxiety , depression and sleep disturbances in HIV + patients chronically treated with an efavirenz-based regimen. 2017;3(2):1-7. 
21. Haas DW, Ribaudo HJ, Kim RB, et al. Pharmacogenetics of efavirenz and central nervous system side effects: an Adult AIDS Clinical Trials Group study. AIDS 2004; 18:2391

22. Drury A, Gleadow-ware S, Gilfillan S, Ahrens J. HIV and mental illness in Malawi and the neuropsychiatric sequelae of efavirenz. 2018;30(March):40-5.

23. Abah IO, Akanbi M, Abah ME, Finangwai Al, Dady CW. Original article Incidence and predictors of adverse drug events in an African cohort of HIV-infected adults treated with efavirenz. 2015;5(September):83-91.

24. Kappelhoff BS, van Leth F, Robinson PA, MacGregor TR, Baraldi E, Montella F, Uip DE, Thompson MA, Russell DB, Lange JM, Beijnen JH. Are adverse events of nevirapine and efavirenz related to plasma concentrations. Antivir Ther. 2005 Jan 1;10(4):489-98.

25. Gaida R, Truter I, Grobler C, Kotze T, Godman B. A review of trials investigating efavirenz-induced neuropsychiatric side effects and the implications. Expert review of anti-infective therapy. $2016 \mathrm{Apr}$ 2;14(4):377-88..

26. Xu B, Guo L, Lee S, Dong Q, Tan Y, Yao H, et al. Genetic variability of CYP2B6 polymorphisms in four southern Chinese populations. 2007;13(14):2100-3.

27. Sarfo FS, Sarfo MA, Chadwick D. Incidence and risk factors for neuropsychiatric events among Ghanaian HIV patients on long-term non-nucleoside reverse transcriptase inhibitor-based therapy. eNeurologicalSci. 2016 Jun 1;3:21-5.

28. Mazhude C, Jones S, Murad S, Taylor C, Easterbrook P. Female sex but not ethnicity is a strong predictor of non-nucleoside reverse transcriptase inhibitor-induced rash. Aids. $2002 \mathrm{Jul}$ 26;16(11):1566-8.

29. Kindie E, Anteneh ZA, Worku E. Time to development of adverse drug reactions and associated factors among adult HIV positive patients on antiretroviral treatment in Bahir Dar City, Northwest Ethiopia. PloS one. 2017 Dec 21;12(12):e0189

30. Abah, Isaac Okoh. "Adverse drug reactions to Antiretroviral drugs: Effect on virologic faliure in a Nigerian cohort of HIV-infected adults on first-line Antiretroviral therapy." (2017).

1. Minstry of Health. National guidelines for comprehensive hiv prevention , care and treatment. 2017; Accesssed at https://aidsfree.usaid.gov/sites/default/files/resources/ethiopia_art_guidelines_2017

2. Masenyetse LJ, Manda SO, Mwambi HG. An assessment of adverse drug reactions among HIV positive patients receiving antiretroviral treatment in South Africa. AIDS research and therapy. 2015 Dec;12(1):6.

3. Agu K, Ochei U, Oparah A, Onoh O. Treatment outcomes in patients receiving combination antiretroviral therapy in Central Hospital, Benin City, Nigeria. Tropical Journal of Pharmaceutical Research. 2010; 9 (1).

4. Agu KA, RC K, MA I, PG I. Medication Adherence and Risk factors for non-adherence among patients taking Highly Active Antiretroviral Therapy. West African Journal of Pharmacy. 2011 Mar 13; 22 (1). 
5. Li H, Marley G, Ma W, Wei C, Lackey M, Ma Q, Renaud F, Vitoria M, Beanland R, Doherty M, Tucker JD. The role of ARV associated adverse drug reactions in influencing adherence among HIV-infected individuals: a systematic review and qualitative meta-synthesis. AIDS and Behavior. $2017 \mathrm{Feb}$ 1;21(2):341-51.

6. Mazzotta E, Agostinone A, Rosso R, Di Biagio A, De Socio GV, Cappelletti A, Zicolella R, Polilli E, Bonfanti P, Di Matteo L, Manzoli L. Osteonecrosis in human immunodeficiency virus (HIV)-infected patients: a multicentric case-control study. Journal of bone and mineral metabolism. 2011 May 1;29(3):383-8.

7. Aberg JA. JIAPAC. Lo I. Notes \& Comments. 2003;160-1.

8. Marzolini C, Telenti A, Decosterd LA, Greub G, Biollaz J, Buclin T. Efavirenz plasma levels can predict treatment failure and central nervous system side effects in HIV-1-infected patients. Aids. 2001 Jan 5;15(1):71-5.

9. Adkins JC, Noble S. Efavirenz. Drugs. 1998 Dec 1;56(6):1055-64. https://doi.org/10.2165/00003495199856060-00014

10. Joint UN. Programme on HIV/AIDS. Global AIDS Update 2016. Joint United Nations Programme on HIV. AIDS. 2016.

11. Teklay G, Legesse B, Legesse M (2013) Adverse Effects and Regimen Switch among Patients on Antiretroviral Treatment in a Resource Limited Setting in Ethiopia. J Pharmacovigilance 1: 115. doi:10.4172/2329-6887.1000115

12. IJBCP International Journal of Basic \& Clinical Pharmacology Assessment of ART adverse reactions and determinants at primary hospital in Ethiopia. 2013;2(2):208-15.

13. Kenedi CA, Goforth HW. A systematic review of the psychiatric side-effects of efavirenz. AIDS and Behavior. 2011 Nov 1;15(8):1803-18.

14. Fumaz CR, Munoz-Moreno JA, Moltó J, Negredo E, Ferrer MJ, Sirera G, Pérez-Alvarez N, Gómez G, Burger D, Clotet B. Long-term neuropsychiatric disorders on efavirenz-based approaches: quality of life, psychologic issues, and adherence. JAIDS Journal of Acquired Immune Deficiency Syndromes. 2005 Apr 15;38(5):560-5.

15. Padilla S, Anto R, Masia M, Martı A. Prediction of Neuropsychiatric Adverse Events Associated with Long-Term Efavirenz Therapy , Using Plasma Drug Level Monitoring. 2018;41(May):0-5.

16. Wambura CA. Assessment of the Prevalence of Central Nervous System Adverse Reactions in adult patients taking Efavirenz based regimens at Mbagathi District Hospital's comprehensive Care Centre (Doctoral dissertation, JKUAT).2015;

17. Abers MS, Shandera WX, Kass JS. Neurological and psychiatric adverse effects of antiretroviral drugs. CNS drugs. 2014 Feb 1;28(2):131-45.

18. Mehta U, Durrheim DN, Blockman M, Kredo T, Gounden R, Barnes KI. Adverse drug reactions in adult medical inpatients in a South African hospital serving a community with a high HIV/AIDS prevalence: prospective observational study. British journal of clinical pharmacology. 2008 Mar;65(3):396-406. 
19. Mueller TE, Ellwanger JH, Michita RT, Matte MC, Renner JD. CYP2B6 516 G> T polymorphism and side effects of the central nervous system in HIV-positive individuals under Efavirenz treatment: Study of a sample from southern Brazil. Anais da Academia Brasileira de Ciências. 2017 May;89(1):497-504.

20. Celesia BM, Sofia SA, Rapisarda L, Maresca M, Vinci L, Gussio M, et al. Anxiety , depression and sleep disturbances in HIV + patients chronically treated with an efavirenz-based regimen. 2017;3(2):1-7.

21. Haas DW, Ribaudo HJ, Kim RB, et al. Pharmacogenetics of efavirenz and central nervous system side effects: an Adult AIDS Clinical Trials Group study. AIDS 2004; 18:2391

22. Drury A, Gleadow-ware S, Gilfillan S, Ahrens J. HIV and mental illness in Malawi and the neuropsychiatric sequelae of efavirenz. 2018;30(March):40-5.

23. Abah IO, Akanbi M, Abah ME, Finangwai Al, Dady CW. Original article Incidence and predictors of adverse drug events in an African cohort of HIV-infected adults treated with efavirenz. 2015;5(September):83-91.

24. Kappelhoff BS, van Leth F, Robinson PA, MacGregor TR, Baraldi E, Montella F, Uip DE, Thompson MA, Russell DB, Lange JM, Beijnen JH. Are adverse events of nevirapine and efavirenz related to plasma concentrations. Antivir Ther. 2005 Jan 1;10(4):489-98.

25. Gaida R, Truter I, Grobler C, Kotze T, Godman B. A review of trials investigating efavirenz-induced neuropsychiatric side effects and the implications. Expert review of anti-infective therapy. $2016 \mathrm{Apr}$ 2;14(4):377-88..

26. Xu B, Guo L, Lee S, Dong Q, Tan Y, Yao H, et al. Genetic variability of CYP2B6 polymorphisms in four southern Chinese populations. 2007;13(14):2100-3.

27. Sarfo FS, Sarfo MA, Chadwick D. Incidence and risk factors for neuropsychiatric events among Ghanaian HIV patients on long-term non-nucleoside reverse transcriptase inhibitor-based therapy. eNeurologicalSci. 2016 Jun 1;3:21-5.

28. Mazhude C, Jones S, Murad S, Taylor C, Easterbrook P. Female sex but not ethnicity is a strong predictor of non-nucleoside reverse transcriptase inhibitor-induced rash. Aids. 2002 Jul 26;16(11):1566-8.

29. Kindie E, Anteneh ZA, Worku E. Time to development of adverse drug reactions and associated factors among adult HIV positive patients on antiretroviral treatment in Bahir Dar City, Northwest Ethiopia. PloS one. 2017 Dec 21;12(12):e0189

30. Abah, Isaac Okoh. "Adverse drug reactions to Antiretroviral drugs: Effect on virologic faliure in a Nigerian cohort of HIV-infected adults on first-line Antiretroviral therapy." (2017).

\section{Tables}

Due to technical limitations, Tables 1-3 are provided in the Supplementary Files section. 
Figures

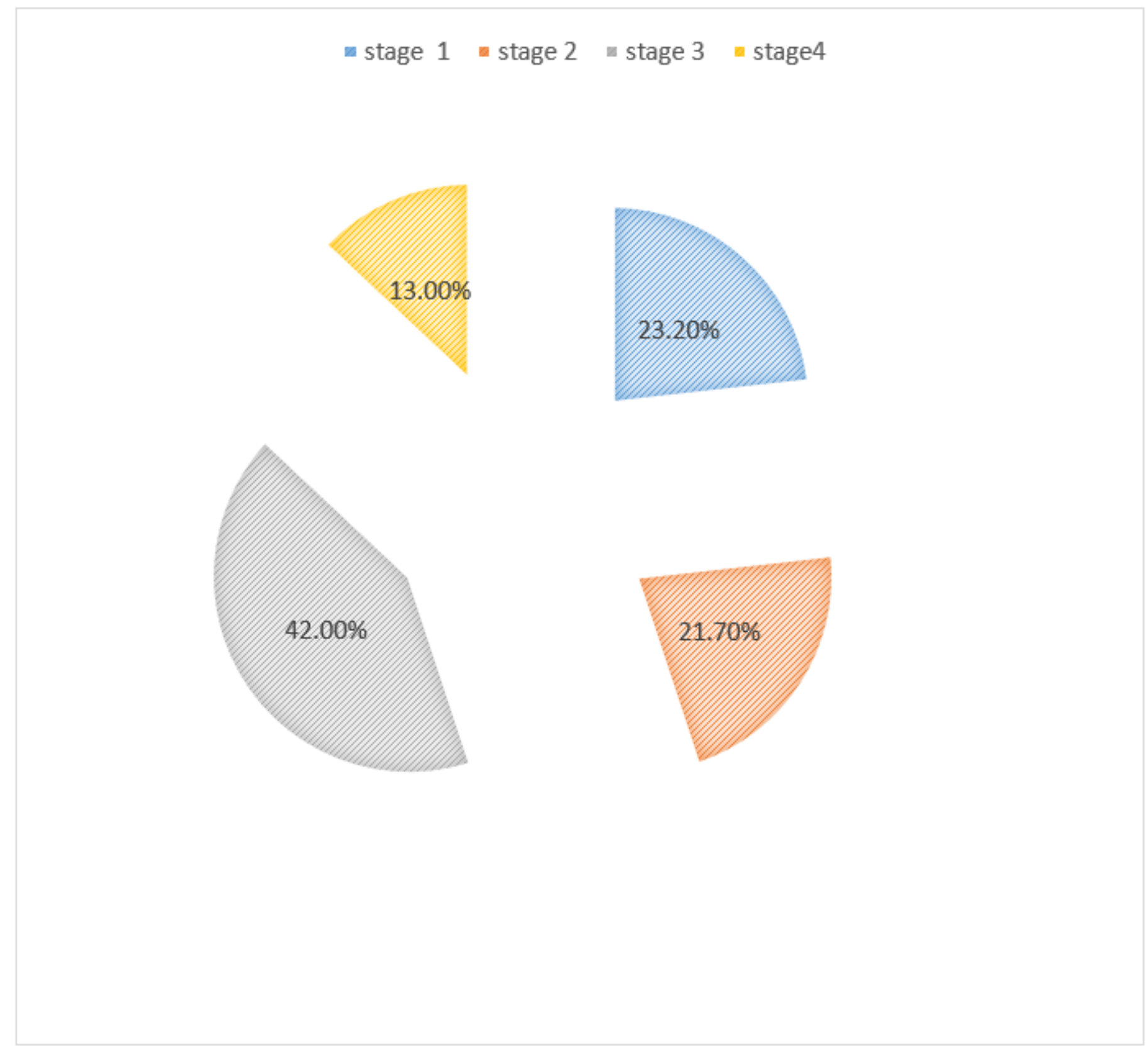

Figure 1

Occurrence of CNS adverse effect and RVI stage of participants attending ART clinic at UOGCSH 2018 $(\mathrm{N}=182)$

\section{Supplementary Files}

This is a list of supplementary files associated with this preprint. Click to download. 
- TableEFV.docx

Page 16/16 